\title{
Polarization in the Cosmic Microwave Background
}

\author{
James G. Bartlett \\ APC, 11 pl. Marcelin Berthelot, 75231 Paris Cedex 05, FRANCE and \\ (UMR 7164 CNRS, Université Paris 7, CEA, Observatoire de Paris)
}

Received on 15 May, 2006

\begin{abstract}
Cosmic microwave background (CMB) studies underpin our understanding of the universe and its history. Until recently, we have relied principally on CMB temperature observations to build our standard cosmological model, but today the field forges ahead into its next frontier - CMB polarization anistropy. Polarization measurements will furnish fresh and independent information on the primordial density perturbations and cosmological parameters, and they offer the exciting potential to detect primordial gravity waves, constrain dark energy and measure the neutrino mass scale. I review the science and long-term goals of CMB polarization measurements and discuss current results and future observational projects. A vigorous program of ground-based, suborbital and space-based (e.g., WMAP and Planck [2008]) experiments is guiding us towards a future space mission dedicated to high precision polarization measurements.
\end{abstract}

Keywords: Cosmic microwave background theory; CMB observations

\section{INTRODUCTION}

Observations of the cosmic microwave background (CMB) anisotropy have driven the remarkable advance of cosmology over the past decade [1]. From them we have learned that we live in a spatially flat universe where structures form by the gravitational evolution of nearly scale invariant, adiabatic perturbations in a predominant form of non-baryonic cold dark matter. Combined with either results from supernovae Ia (SNIa) distance measurements [2], the determination of the Hubble constant [3] or measures of large scale structure [4], they furthermore demonstrate that a mysterious dark energy (cosmological constant, vacuum energy, quintessence...) dominates the total energy density of our Universe. These observations have established what is routinely called the standard cosmological model: $\Omega_{\mathrm{M}} \approx 0.25=$ $1-\Omega_{\Lambda}, \Omega_{\mathrm{B}} h^{2} \approx 0.022$ and $H_{0} \approx 70 \mathrm{~km} / \mathrm{s} / \mathrm{Mpc}$ [5]. Because the observations in fact over-constrain the model, they test its coherence and its foundations, marking a new era in cosmology.

The CMB results are remarkable for a number of reasons. They divulge the existence of density perturbations on superhorizon scales at decoupling and hence give us a glimpse of new physics (inflation or other) working in the early universe. The observed peaks in the power spectrum affirm the key idea that coherent density perturbations enter the horizon and begin to oscillate as acoustic waves in the primordial plasma prior to recombination; their position justifies the long-standing theoretical preference for flat space with zero curvature. Their heights measure both the total matter and baryonic matter densities and thereby attest that most of the matter is non-baryonic; and, in a scientific tour de force, the CMB-determined baryon density broadly agrees with the totally independent estimation from Big Bang Nucleosynthesis [6].

These milestones are founded almost exclusively on study of the temperature, or total intensity, anisotropies. The Planck mission $^{1}$ (launch 2008) will essentially complete this work by decade's end with foreground-limited temperature maps down to $\sim 5$ arcmin resolution, leaving only the smallest scales unexplored. In this exciting context, the field is already turning to CMB polarization measurements for their wealth of new information.

\section{CMB POLARIZATION}

Humble Thomson scattering generates CMB polarization at decoupling [7], because of the polarization dependence of the differential cross section: $d \sigma / d \Omega \propto\left|\varepsilon^{\prime} \cdot \varepsilon\right|^{2}$, where $\varepsilon$ and $\varepsilon^{\prime}$ are the incoming and outgoing polarization states [8]. Note that only linear polarization is involved. This dependence on polarization state means that an observer measuring a given polarization sees light scattered preferentially from certain directions around the scattering electron in the last scatter surface. The orthogonal polarization preferentially samples different parts of the sky. Any local intensity anisotropy around the scattering electron thus creates a net linear polarization at the observer's detector. Quantitatively, it is the local quadrupolar temperature anisotropy that produces the net polarization, because of the $\cos ^{2} \theta$ dependence of the cross section. Also observe that the signal is actually generated in the last scattering surface, where the optical depth transits from large to small values; the optical depth must of course be non-zero, but too large a value would erase any local anisotropy around the scattering electrons.

Polarized light is commonly described using the Stokes parameters [8]. Since the CMB is linearly polarized, we only need the Stokes parameters $Q$ and $U$, each of which is defined as the intensity difference between two orthogonal polarization directions. Let $(x, y)$ and $\left(x^{\prime}, y^{\prime}\right)$ refer to two coordinate systems situated perpendicular to the light propagation direc-

\footnotetext{
1 http://www.esa.int/science/planck
} 

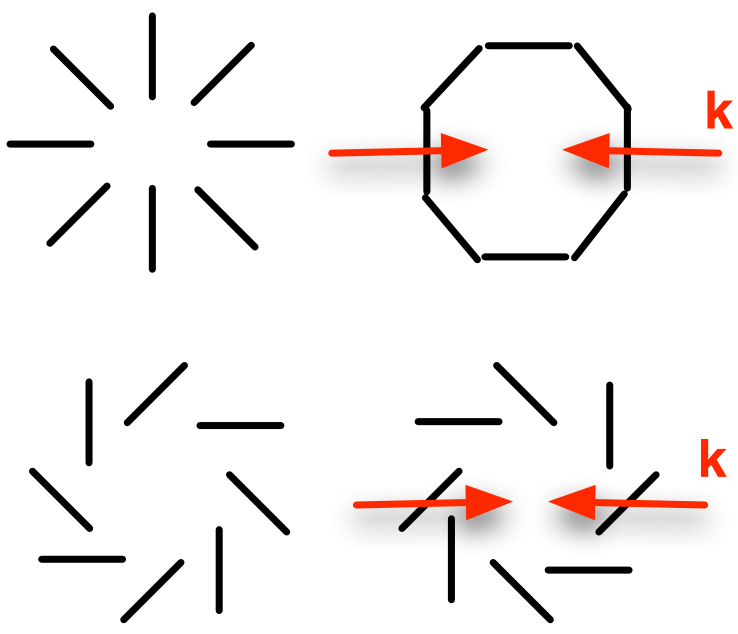

FIG. 1: Polarization patterns around an local intensity extremum. Black bars designate the polarization direction. The upper row depicts the even parity $E$-mode (negative on the left, positive on the right), and the lower row the odd parity $B$-mode (negative on the left, positive on the right). The red arrows show the projected wave vectors of plane wave perturbations converging at the extremum.

tion and rotated by 45 degrees with respect to each other. Then $Q \equiv I_{y}-I_{x}$ and $U \equiv I_{y^{\prime}}-I_{x^{\prime}}$.

Clearly, the values of $Q$ and $U$ depend on the orientation of the coordinate system used at each point on the sky. It is convenient from a theoretical standpoint to look for coordinatefree descriptions, which could then be translated into any particular coordinate system. Two such descriptions were first proposed for the CMB by Zaldarriaga \& Seljak and by Kamionkowski et al. [9]. The former, in particular, model polarization as a spin 2 field on the sphere, an approach used in the publicly available CMB codes [10].

The coordinate-free descriptions distinguish two kinds of linear polarization pattern on the sky by their different parities. In the spinor approach, the even parity pattern is called the $E$-mode and the odd parity pattern the $B$-mode. Consider a peak in CMB intensity (see Figure 1). If the polarization bars are oriented either in a tangential or a radial pattern around the peak, we have an $E$-mode; if, on the other hand, they are oriented at 45 degrees (relative to rays emanating from the peak), we have a $B$-mode: a reflection of the sky about any line through the peak leaves the $E$-mode unchanged (even parity), while the $B$-mode changes sign (odd parity) ${ }^{2}$.

Another useful way to see this is to consider the wave vectors of the plane wave perturbations making up the intensity peak; they radially point towards the peak center (see Figure 1). We then see that an $E$-mode plane wave has its polarization either perpendicular or parallel to the wave vector. A $B$-mode plane wave, on the other hand, has a linear polariza-

\footnotetext{
2 These local considerations generalize to the sphere [9].
}

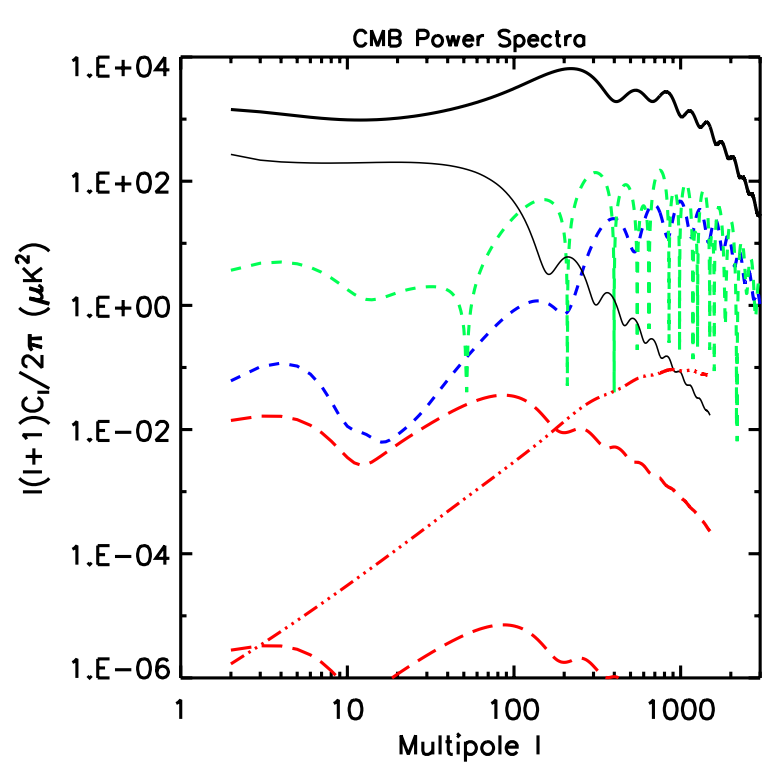

FIG. 2: Angular power spectra. The bold solid black line shows the temperature power spectrum from scalar perturbations in the standard flat model (WMAP 3year values were adopted: $\quad\left(\Omega_{\mathrm{M}} h^{2}, \Omega_{\mathrm{B}} h^{2}, h, n_{\mathrm{s}}, \tau, \sigma_{8}\right)=$ $(0.127,0.0223,0.73,0.951,0.09,0.74)[15])$, while the thin black line gives the temperature perturbations from tensor perturbations when $r=0.5$. The green (upper) and blue (lower) short dashed curves are, respectively, the scalar $T E$ (absolute value shown) and $E E$ power spectra for the standard model; the former is well measured on large scales by WMAP [26]. The red long dashed lines indicate the tensor $B$-mode power for $r=0.5$ (upper) and $r=10^{-4}$ (lower). Gravitational lensing produces the $B$-mode power shown as the red 3-dot-dashed curve peaking at $l \sim 1000$.

tion at 45 degrees to the wave vector. The wave vector in fact defines a natural coordinate system for definition of the Stokes parameters: in this system, $Q=E$ and $U=B$. This is particularly useful when discussing interferometric observations.

\section{A. The Physical Content of CMB Polarization}

This decomposition of polarization into $E$ and $B$-modes is powerful and practical. Firstly, the two different modes are generated by different physical mechanisms, which is not surprising, since they are distinguished by their parity. Secondly, their different parity guaranties that we can separate and individually measure the two modes and total intensity patterns on the sky. This is extremely important because the three signals vary greatly in amplitude (see Figure 2).

Since theory predicts Gaussian perturbations (of zero mean), and current observations remain fully consistent with this expectation, we describe CMB anisotropy with the power spectrum $C_{l}$, which is nothing other than the second moment of the perturbation field in harmonic space (i.e., the variance). Most CMB milestones to date have been obtained from tem- 
perature measurements, which in this context means from measurement of the temperature angular power spectrum $C_{l}^{\mathrm{TT}}$. When considering polarization, however, there are in fact 4 power spectra to determine: $C^{\mathrm{TT}}, C^{\mathrm{TE}}, C^{\mathrm{EE}}, C^{\mathrm{BB}}$. We do not expect the primordial perturbations to care about parity, which implies that statistical measures of the anisotropy, such as power spectra, should have even parity. This means that the other two possible power spectra $C_{l}^{\mathrm{TB}}=C^{\mathrm{EB}}=0^{3}$.

Inflation generates both scalar (S) density perturbations [11] and tensor (T) gravity wave perturbations [12]. The scalar perturbations are created by quantum fluctuations in the particle field (usually assumed to be a scalar field) driving inflation. After inflation, these perturbations grow by gravity to form galaxies and the observed large scale structure. Gravity waves, on the other hand, decay once they enter the horizon, and thus leave their imprint in the CMB on large angular scales (around and larger than the decoupling horizon, $\sim 1 \mathrm{deg}$ ).

Gravity wave production by inflation, although a reasonable extrapolation of known physics, would nevertheless be something fundamentally new. These waves would not be generated by any classical or even quantum source (i.e., by the right-hand-side of Einstein's equations); we suppose instead that the gravitational field itself (more specifically, the two independent polarization states of a free gravity wave) experiences vacuum quantum fluctuations like any field. Finding tensor perturvations from inflation would therefore not simply be a detection of gravity waves, but also a remarkable observation of the semi-classical behavior of gravity.

Current data limit (see below) the tensor contribution to the temperature power spectrum to less than $50 \%$, and it could be much less. We quantify the relative amplitude of the scalar and tensor perturbations by the parameter $r \equiv \mathcal{P}_{\mathrm{T}} / \mathcal{P}_{\mathrm{S}}$, where $\mathcal{P}_{\mathrm{T}}$ and $\mathcal{P}_{\mathrm{S}}$ represent the power in the respective modes at a pivot wavenumber ${ }^{4}$ [13]. Since the scalar power is measured, we use $r$ to express the gravity wave amplitude. The first year WMAP data combined with large-scale structure observations limited $r<0.53$ (95\%) [14]; improving on this, the third year WMAP data alone limit $r<0.55$ (95\%) for powerlaw primordial spectra [15]). Unlike the scalar modes, which depend on the slope of the inflation potential, the gravity wave amplitude depends only on the energy scale of inflation, $E_{\mathrm{I}}$ (specifically, $\mathcal{P}_{\mathrm{T}} \propto\left(E_{\mathrm{I}} / M_{\mathrm{pl}}\right)^{4}$, where $M_{\mathrm{pl}}$ is the Planck mass). Quantitatively $^{5}$, we have $E_{\mathrm{I}}=3.4 \times 10^{16} \mathrm{GeV} r^{1 / 4}$. Thus, the above limit on $r$ corresponds to an upper limit on the inflation scale of $E_{I}<2.8 \times 10^{16} \mathrm{GeV}$.

\footnotetext{
3 This does not hold for foreground emission

${ }^{4}$ In $[14,15]$, they use $k=0.002 \mathrm{Mpc}^{-1}$.

5 The numerical relation refers to the definition of $r$ used in the above references and corresponds to the parameter employed in the CAMB code; it furthermore adopts the $2 \sigma$ upper limit on the scalar power amplitude given by WMAP-3 [15].
}

\section{B. The Importance of the $E-B$ Decomposition}

Both scalar and tensor perturbations generate temperature anisotropy $\left(C^{\mathrm{TT}}\right)$; primordial scalar perturbations, however, cannot create $B$-mode polarization, and hence only contribute to $C^{\mathrm{EE}}$ and $C^{\mathrm{TE}}$. We can understand this by considering a plane wave scalar perturbation passing over a scattering electron: the local intensity quadrupole around the electron must be aligned with the wave vector, which implies that the polarization of the scattered light must be either perpendicular or parallel to the projected wave vector - in other words, a pure $E$ mode. The axial symmetry imposed by the scalar nature of the density perturbations prevents any $B$-mode production.

Figure 2 shows inflationary predictions for the various CMB power spectra from inflation-generated scalar and tensor perturbations in the standard flat model with WMAP-3 parameter values $\left(\Omega_{\mathrm{M}} h^{2}, \Omega_{\mathrm{B}} h^{2}, h, n_{\mathrm{s}}, \tau, \sigma_{8}\right)=$ $(0.127,0.0223,0.73,0.951,0.09,0.74)^{6}$ [15]. The temperature power spectrum from scalar perturbations is given by the bold solid black curve, while the light, black solid curve gives the maximum allowable tensor contribution to the temperature power spectrum, i.e., at the current limit of $r<0.53$. It would be very hard to significantly improve on this limit with temperature measurements alone. One can do better by combining CMB temperature and large-scale structure data; Spergel et al. [15], for example, tighten the limit to $r<0.28$ (95\%) by combining the WMAP-3 data with the measured SDSS power spectrum. While powerful, this approach is indirect and model-dependent. Fortunately, CMB polarization offers a direct and much more sensitive gauge for the existence of primordial gravity waves, which explains the intense interest in CMB polarization science.

The other curves in the figure correspond to the various polarization power spectra. The short dashed green (upper) and blue (lower) lines show the predicted $C^{\mathrm{TE}}$ and $C^{\mathrm{EE}}$ power spectra generated by scalar perturbations. The TE cross spectrum in fact changes sign, but I only plot its absolute value. The bump at low multipoles in the polarization spectra arises from Thomson scattering after reinonization; note that I have taken an optical depth of $\tau=0.09$ [15].

These predictions for the scalar generated polarization spectra follow directly from the measured temperature power spectrum and the assumption - usually adopted in the standard model - that the scalar perturbations are purely adiabatic, as befits most simple inflationary scenarios. Given the measured temperature spectrum, we could change the predicted $C^{\mathrm{TE}}$ and $C^{\mathrm{EE}}$ spectra by adding a mixture of isocurvature perturbations. Observations of these polarization modes therefore constrain the presence of such isocurvature modes ${ }^{7}$.

In contrast to scalar perturbations, gravity waves $(\mathrm{T})$ push and pull matter in directions perpendicular to their propagation, aligning the local intensity quadrupole in the plane perpendicular to the wave vector. The loss of axial symmetry

\footnotetext{
${ }^{6}$ These calculations where made using CAMB (http://www. camb.info )

7 These modes are also constrained by large scale structure observations.
} 
permits both $E$ and $B$-mode production. Since the expansion dampens gravity waves on scales smaller than the horizon, these tensor effects only appear on angular scales larger than $\sim 1^{\circ}$ (the angular size of the decoupling horizon). Hence, $B-$ mode polarization on large angular scales is the unique signature of primordial gravity waves [17].

The amplitude of the gravity wave signal depends only on the energy scale of inflation, and a measurement of $B$-mode polarization on large scales would therefore give us a direct determination of the energy scale of inflation ${ }^{8}$. The red longdashed curves in Figure 2 show the tensor $B$-mode spectrum for two different amplitudes - the upper curve for the the current limit of $r<0.53\left(E_{\mathrm{I}} \sim 3 \times 10^{16} \mathrm{GeV}\right)$, and the lower one for $r=10^{-4}\left(E_{\mathrm{I}} \sim 3.4 \times 10^{15} \mathrm{GeV}\right)$.

\section{Gravitational Lensing}

Gravitational lensing of $\mathrm{CMB}$ anisotropy by structures forming along the line-of-sight to decoupling also generates $B$-mode polarization, but on smaller scales [18]. Lensing deviates the photon trajectories (preserving surface brightness) and scrambles our view of the decoupling surface [19]. As the $E-B$ modes are defined as pure parity patterns on the sky, scrambling any such pattern will clearly destroy its pure parity, thereby leaking power into the opposite parity mode. If, for example, there were only $E$-mode perturbations at decoupling (e.g., gravity waves are negligible), we would still see some $B$-mode in our sky maps on small angular scales caused by gravitational lensing.

This lensing signal has its virtues and sins in the present context. On the down side, it masks the gravity wave $B$-mode with a foreground signal with an identical electromagnetic spectrum; thus, we cannot remove it using frequency information. We can, however, extract and remove the lensing signal by exploiting the unique mode-mode coupling (between different multipoles, absent in the primary anisotropies) induced by the lensing [20]. Assuming that we eventually control foreground contamination sufficiently well, uncertainty in this cleaning process would ultimately limit our sensitivity to gravity waves [21].

On the positive side, the lensing signal carries important information about the matter power spectrum and its evolution over a range of redshift inaccessible to other observations. This gives us a powerful means of constraining dark energy and a singular method for determining the neutrino mass scale [22]. Since the expansion governs the matter perturbation growth rate, comparison of the amplitude of the power spectrum at high redshift to its amplitude today probes the influence of dark energy. The shape of the power spectrum, on the other hand, is affected by the presence of massive neutrinos, which tend to smooth out perturbations on small scales by free streaming.

\footnotetext{
${ }^{8}$ More precisely, at the end of inflation.
}

Recent studies indicate that by measuring the lensing polarization signal to its cosmic variance limit, we would obtain a $1 \sigma$ sensitivity to the sum of the 3 neutrino masses of $\sigma_{\Sigma}=0.035 \mathrm{eV}$ [22]. This is extremely important: current neutrino oscillation data call for $\mathrm{a}^{9} \Delta m^{2}=\left(2.4_{-0.6}^{+0.5}\right) \times 10^{-3} \mathrm{eV}^{2}$ $(2 \sigma)$ [23], implying that the summed mass of the three neutrino species exceeds the ultimate $\mathrm{CMB}$ sensitivity. CMB polarization therefore provides a powerful and unique way to measure the neutrino mass scale, down to values unattainable in the laboratory.

The red triple-dot-dashed curve in Figure 2 shows the $B$ mode polarization predicted from lensing in this model. Its amplitude is set by the amplitude of the primordial $E$-mode signal and of the matter power spectrum as it evolves. Since gravity waves generate both $E$-modes (their contribution is not shown in the figure) and $B$-modes of roughly equal power, we expect the scalar $E$-mode to dominate. Thus, we have a good idea of the overall amplitude of the lensing $B$-mode spectrum, although the exact amplitude and shape will depend, as discussed, on the presence of isocurvature modes, neutrinos and the nature of dark energy. For the curve shown in Figure 2, I have adopted the standard model (no isocurvature perturbations) with a pure cosmological constant and have ignored neutrinos.

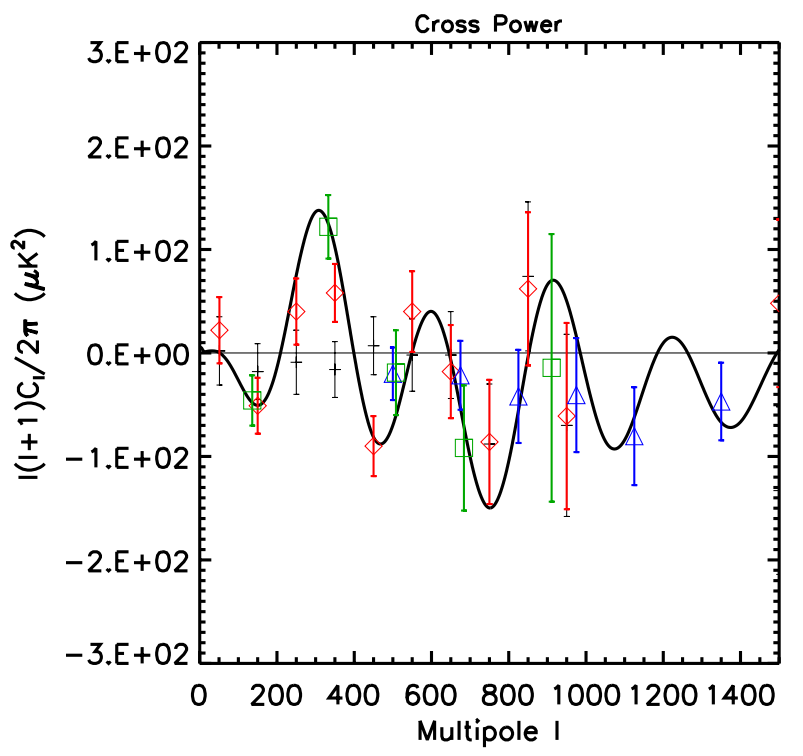

FIG. 3: TE power spectra. The curve shows the power predicted by the standard model (and measured on large scales by WMAP [26], although not reproduced here). Red diamonds give the BOOMERanG results, green boxes the DASI 3-year results and blue triangles the CBI results. The thin black error bars show the BOOMERanG $T B$ power, a foreground tracer.

\footnotetext{
${ }^{9}$ Here, $\Delta m^{2}$ is the difference between the singlet neutrino mass squared and the mean squared mass of the neutrino doublet; see [22].
} 


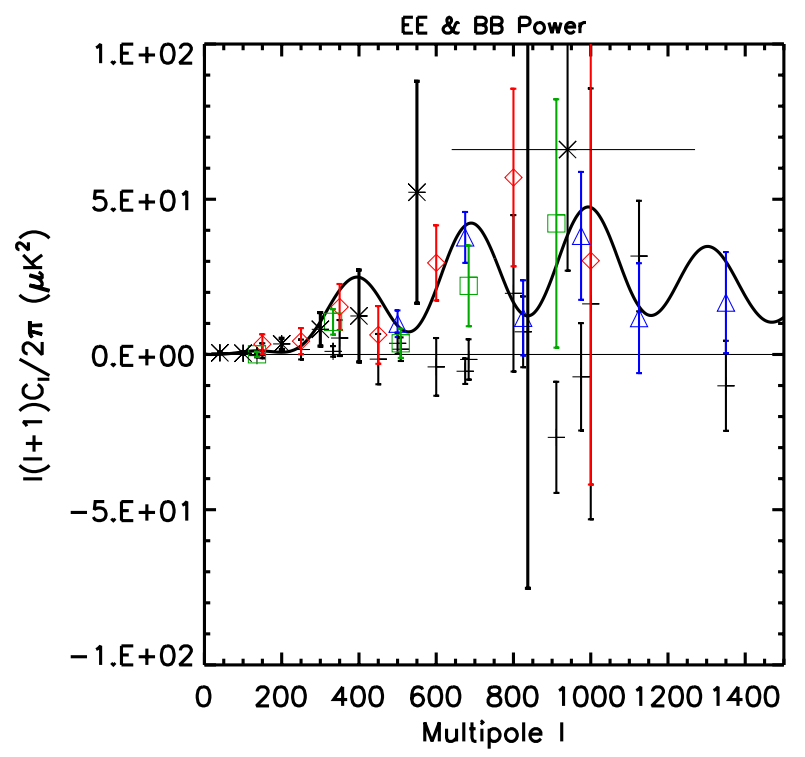

FIG. 4: EE and BB power spectra. The curve shows the standard model prediction for $C^{\mathrm{EE}}$. Points are labeled as in the previous figure. The black asterisk is the CAPMAP $E$-mode power measurement, and the black $X$ 's show the WMAP-3 EE spectrum for $l>20$ (the pre-cleaned QWV row taken from Table 8 of Page et al. [26]. In this figure, the thin black error bars give each experiment's $B$-mode measurements (excluding WMAP); they are all consistent with zero.

\section{OBSERVATIONAL EFFORT}

Although we often refer to polarization as the next step in $\mathrm{CMB}$ science, this erroneously gives the impression that it remains for the future, when in fact different experiments have already measured polarization on a variety of angular scales. I give a summary of some of these results in Figures 3 and 4.

The DASI experiment at the South Pole was the first to detect CMB polarization, both $E$ and $T E$ modes [24]; their recently published 3-year results [25] are shown in Figures 3 and 4 as the green squares. The original DASI detection was followed by WMAP's measurement of $C^{\mathrm{TE}}$ on large scales down to $l \sim 500$ from the first year data [26]; these are not reproduced in the figure.

The BOOMERanG collaboration has reported measurements of $C^{\mathrm{TT}}, C^{\mathrm{TE}}$ and $C^{\mathrm{EE}}$ and a non-detection of $B$ modes [27]. Combining their new BOOMERanG data with other $\mathrm{CMB}$ and large scale structure data, MacTavish et al. [28] constrain $r<0.36(95 \%)$. These results are shown in Figures 3 and 4 as the red diamonds.

The CBI experiment has also published new measurements of $C^{\mathrm{TT}}, C^{\mathrm{TE}}$ and $C^{\mathrm{EE}}$, as well as a non-detection of $B-$ modes [29]. These are shown in Figures 3 and 4 as the blue triangles. The black asterisk in Figure 4 gives the $E$-mode power measurement by CAPMAP [30].

Finally, WMAP has just published its 3-year data set and analysis, for which Page et al. [26] present the polarization results; I show the measured $E$-mode power spectrum as the $X$ 's in Figure 4. Besides these measurements of the primordial spectrum, WMAP has given us important information about the Galactic foregrounds. On large scales (low multipoles), polarized foreground emission dominates the cosmic $E E$ (and $B B$ ) signal even in the foreground minimum at $\mathrm{v} \sim 60 \mathrm{GHz}$ and at high Galactic latitudes. This situation is in contrast to that of the temperature anisotropies, which dominate the sky at high Galactic latitudes over a rather large range of frequencies around the minimum ( $\sim 80 \mathrm{GHz}$ for intensity). The foreground power, however, drops off with increasing multipole $l$, so that the cosmic $E E$ signal dominates by $l \sim 100$.

These new WMAP results have considerably tightened the constraint on the optical depth to reionization. The full model fit to the $T T, T E$ and $E E$ spectra now gives $\tau=0.09 \pm 0.03$, a result that is mainly driven by the $E E$ measurement at low multipole (in the "reionization bump"). This in turn improves the constraint on the primordial spectral index $n_{s}$ by significantly reducing the degeneracy between $\tau$ and $n_{s}$. The best fit model favors a a value below the pure scale invariant Harrison-Zel'dovich spectrum [15, 31].

All of these results are consistent with each other and with the prediction, shown in the figures as the black curve, of the standard cosmological model assuming pure adiabatic modes. The measurements are, however, still a long way from the precision we currently enjoy on the temperature power spectrum.

Scheduled for launch in 2007/2008, the Planck satellite will greatly advance our knowledge of CMB polarization by providing foreground/cosmic variance-limited measurements of $C^{\mathrm{TE}}$ and $C^{\mathrm{EE}}$ (and $C^{\mathrm{TT}}$ ) out beyond $l \sim 1000$. We also expect to detect the lensing signal, although with relatively low precision, and could see gravity waves at a level of $r \sim 0.1$. The Planck blue book quantifies these expectations.

A leap in instrument sensitivity is required in order to go beyond Planck and get at the $B$-modes from lensing and gravity waves. This important science is motivating a vast effort world wide at developing a new generation of instruments based on large detector arrays. Numerous ground-based and ballon-borne experiments are actually observing or being prepared. In the longer term future, both NASA (Beyond Einstein) and ESA (Cosmic Vision) have listed a dedicated CMB polarization mission as a priority in the time frame 2015-2020. Such a mission could reach the cosmic variance limit on the lensing power spectrum to measure the neutrino mass scale and perhaps detect primordial gravity waves from inflation near the GUT scale. Conquest of the new frontier has begun.
[1] G. F. Smoot et al., ApJ 396, L1(1992); A.E. Lange et al., Phy. Rev. D 63, 042001 (2001); R. Stompor et al., ApJ 561,
L7 (2001); C. L. Bennett et al., ApJS 148, 1 (2003); D. Scott and G. Smoot, The Review of Particle Physics (Preprint astro- 
ph/0601307); G. Hinshaw et al., Preprint astro-ph/0603451.

[2] R. A. Knop et al., ApJ 598, 102 (2003); A.G. Riess et al., ApJ 607, 665 (2004); P. Astier et al., A\&A, 447, 31 (2006).

[3] W.L. Freedman et al., ApJ 553, 47 (2001).

[4] M. Tegmark et al., ApJ 606, 702 (2004); S. Cole et al., MNRAS 362, 505 (2005).

[5] D. N. Spergel et al., ApJS 148, 175 (2003); U. Seljak et al., Phys. Rev D 71, 103515 (2005); A. G. Sanchez et al., MNRAS 366, 189 (2006); D. N. Spergel et al., Preprint astro$\mathrm{ph} / 0603449$.

[6] G. Steigman, Int. J. Mod. Phys. E15, 1 (2006).

[7] J. R. Bond and G. Efstathiou, ApJ 285, L45 (1984); W. Hu and M. White, New Astron. 2, 323 (1997).

[8] G. B. Rybicki and A. P. Lightman, Radiative processes in astrophysics (New York: Wiley-Interscience, 1979).

[9] M. Zaldarriaga and U. Seljak, Phys. Rev. D 55, 1830 (1997); A. Kamionkowski, A. Kosowsky, and A. Stebbins, Phys. Rev. D 55, 7368 (1997).

[10] U. Seljak and M. Zaldarriaga, ApJ 469, 437 (1996) (http://www.cmbfast.org ); A. Lewis, A. Challinor, and A. Lasenby, ApJ 538, 473 (2000) (http://camb.info ).

[11] V. F. Mukhanov and G.V. Chibisov, JETP Lett. 33, 532 (1981); A.H. Guth and S.-Y. Pi, Phys. Rev. Lett 49, 1110 (1982); S. W. Hawking, Phys. Lett. B 115, 295 (1982); A. A. Starobinsky, Phys. Lett. B 117, 175 (1982); J. M. Bardeen, P. J. Steinhardt, and M. S. Turner, Phys. Rev. D 28, 679 (1983).

[12] A.A. Starobinsky JETP Lett. 30, 682 (1979); A.V. Veryaskin, V.A. Rubakov, and M.V. Sazhin, Soviet Astron. 27, 16 (1983); L.F. Abbott and M. Wise, Nucl. Phys. B 244, 541 (1984); M. Kamionkowski and A. Kosowsky, Ann. Rev. Nucl. Part. Sci. 49 77 (1999).

[13] S. M. Leach, A. R. Liddle, J. Martin, and D.J. Schwarz, Phys. Rev. D 66, 023515 (2002)

[14] H.V. Peiris et al., ApJS 148, 213 (2003).
[15] D.N. Spergel et al., Preprint, astro-ph/0603449.

[16] R. Trotta, Preprint, astro-ph/0608116.

[17] U. Seljak and M. Zaldarriaga, Phys. Rev. Lett. 78, 2054 (1997); M. Kamionkowski, A. Kosowsky, and A. Stebbins, Phys. Rev. Lett. 78, 2058 (1997).

[18] M. Zaldarriaga and U. Seljak, Phys. Rev. D 58, 023003 (1998).

[19] A. Blanchard and J. Schneider, A\&A 184, 1 (1987); U. Seljak, ApJ 463, 1 (1996); F. Bernardeau, A\&A 324, 15 (1997).

[20] W. Hu and T. Okamoto, ApJ 574, 566 (2002); T. Okamoto and W. Hu, Phys. Rev. D 67, 083002 (2003).

[21] M. Kesden, A. Cooray, and M. Kamionkowski, Phys. Rev. Lett. 89, 011304 (2002); L. Knox and Y.-S. Song, Phys. Rev. Lett 89, 011303 (2002); U. Seljak and C.M. Hirata, Phys. Rev. D 69, 043005 (2004).

[22] M. Kaplinghat, K. Lloyd, and Y.-S. Song, Phys. Rev. Lett. 91, 241301 (2003); M. Kaplinghat, New. Astron. Rev. 47, 893 (2003); J. Lesgourgues, L. Perotto, S. Pastor, and M. Piat, Phys. Rev. D 73, 045021 (2006).

[23] G.L. Fogli, E. Lisi, A. Marrone, and A. Palazzo, Preprint hep$\mathrm{ph} / 0506083$.

[24] J.M. Kovac, E.M. Leitch, C. Pryke, J.E. Carlstrom, N.W. Halverson, and W.L. Holzapfel, Nature 420, 772 (2002).

[25] E.M. Leitch, J.M. Kovac, N.W. Halverson, J.E. Carlstrom, C. Pryke, and M.W.E. Smith, ApJ 624, 10 (2005).

[26] A. Kogut et al., ApJS 148, 161 (2003); L. Page et al., Preprint astro-ph/0603450.

[27] F. Piacentini et al., ApJ 647, 833 (2006); T.E. Montroy, et al. ApJ 647, 813 (2006).

[28] C.J. MacTavish et al., ApJ 647, 799 (2006).

[29] J.L. Sievers et al., Preprint astro-ph/0509203.

[30] D. Barkats et al., ApJ 619, L127 (2005).

[31] H.K. Eriksen et al., Preprint astro-ph/0606088. 